\begin{tabular}{|c|c|}
\hline 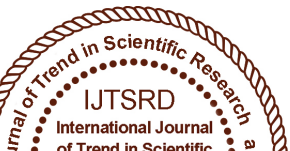 & $\begin{array}{l}\text { International Journal of Trend in Scientific } \\
\text { Research and Development (IJTSRD) }\end{array}$ \\
\hline 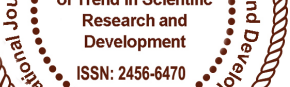 & International Open Access Journal \\
\hline 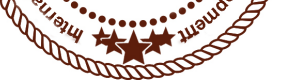 & ISSN No: $2456-6470$ | www.ijtsrd.com | Volume - 2 | Issue - 5 \\
\hline
\end{tabular}

\title{
Analysis of Computer Aided Design Employed in Landscape Design
}

\author{
Uppada Komali ${ }^{1}$, Rajesh CVS ${ }^{2}$ \\ ${ }^{1,2}$ Assistant Professor' Department of Mechanical Engineering, \\ ${ }^{1}$ Avanthi Institute of Engineering and Technology, Vizianagaram, Andhra Pradesh, India \\ ${ }^{2}$ Vizag Institute of Technology, Visakhapatnam, Andhra Pradesh, India
}

\begin{abstract}
This research aims at making a thorough analysis on the computer aided design (CAD) employed in landscape design in an attempt to make computer play a better role in landscaped design and improve the quality of the design. This essay firstly gives a picture of the current development of CAD employed in landscape design, then points out the problems and provides relative solutions. Then it comes to the application of CAD. Judging from the process of CAD landscape design and common CAD software programs, this essay introduces several commonly used software programs and explains the advantages and shortcomings of these programs to allow users to choose according to their needs. Finally, since virtual reality is the latest computer technology, this essay also gives detailed analysis of virtual reality's application to practical use. It explains the process of modeling of virtual reality and gives further demonstration of CAD's advantages in landscape design. Although there is still a lot to be advanced, the trends of CAD are promising. CAD and traditional landscape design are complementary to each other and will make great progress.
\end{abstract}

Keywords: Computer aided design; Landscape design; Virtual reality.

\section{INTRODUCTION}

Computer has gone through tremendous progress since the day it was invented. Now computer has become ubiquitous and achieved great accomplishments in various fields. Designers use computer to design, make plans and simulations, which alleviates their workload, increases efficiency, reduces working time, improves the quality and makes the design fit for the requirements of the client.

However, computer came to China at a relatively late period. There are several problems need to be tackled, such as a disparity in the development of computer. So how to combine CAD in landscape design has become the focus and trend among designers and computer experts, as well as the hot topic in this line of business. In an attempt to address to such problems, this essay aims at demonstrating the advantages of computer by analyzing the actual examples of how CAD assists landscape design.

\section{CURRENT SITUATION OF COMPUTER AIDED DESIGN (CAD)}

1. Requiring interdisciplinary expertise. Landscape design requires a combination of time and space. The design demands not only the display of space or objects, such as plants, but also dynamic nature. Only these two are perfectly combined into the design can the ideas of the designer be shown, which constitute a perfect design. Since landscape design involves many fields, requires enormous amount of information and deals with complicated objects, the current computer technology cannot meet these requirements.

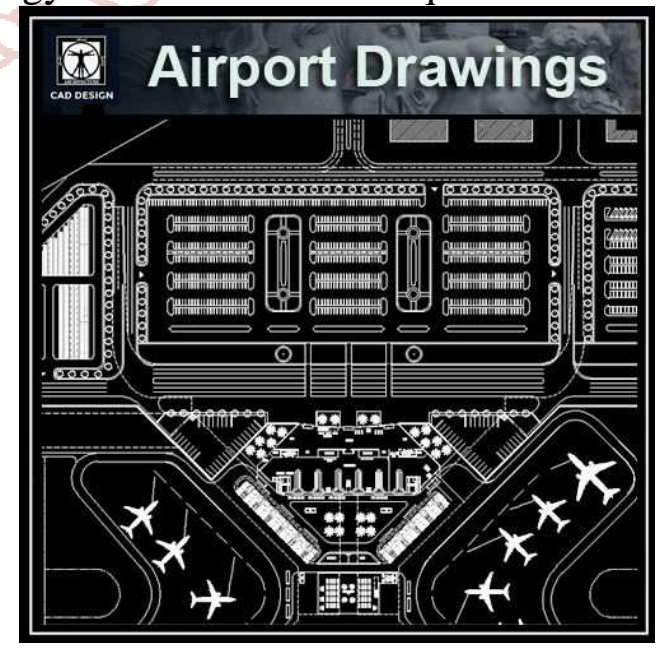


2. Developing at a relatively late period. Computer aided design has become very mature in developed countries in 1980s. While in China, it falls behind. Although great progress has been made, but there is a lot to be advanced and many problems remain to be solved. (3) Having no suitable CAD software programs. Landscape designers have to take extra work to finish the design or part of the design in other ways. $\mathrm{CAD}$ is the main trend, which requires landscape designers to have knowledge about computer technology and software. Many software programs have been developed in an attempt to make it fit for Chinese landscape design, but these software programs are inefficient, of poor quality and not widely used, therefore not able to satisfy the requirements of the designers. And the widely used professional CAD software programs in the market are developed by foreign companies, whose language barrier and high prices make it rather difficult for Chinese landscape designers.

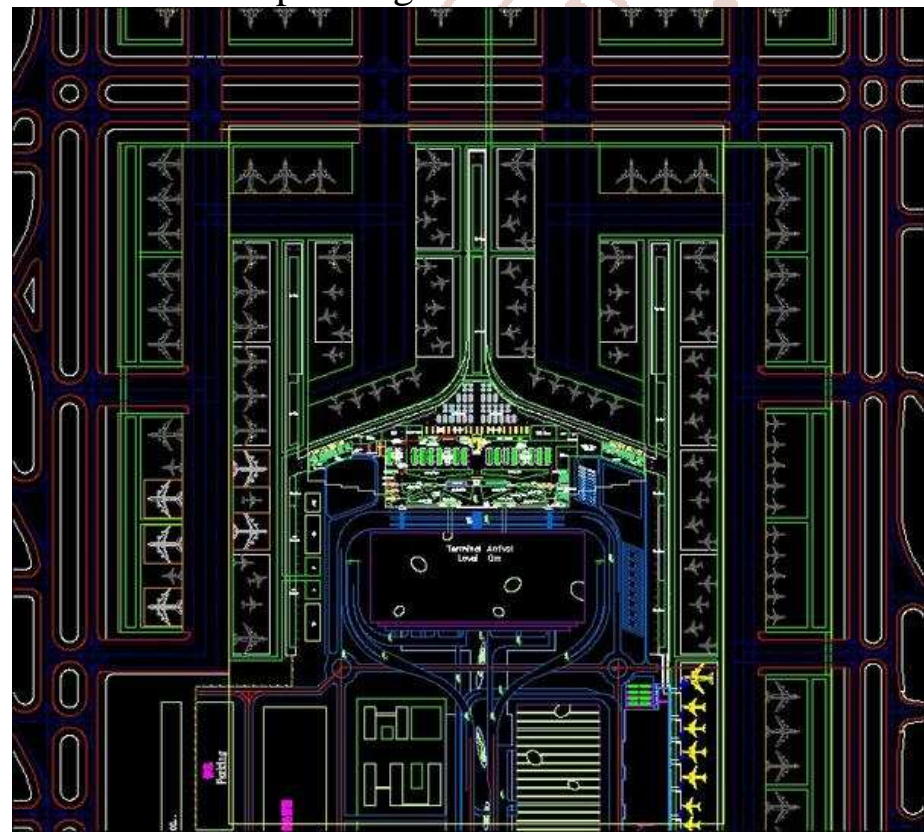

\section{COMPUTER AIDED DESIGN (CAD) EMPLOYED IN LANDSCAPE DESIGN}

\section{The process of CAD landscape design}

CAD landscape design is a combination of computer and design, including creating design assignment, surveying the task, gathering materials, creating preliminary design, producing designs and producing construction drawings. Throughout such processes, the designer needs store the detailed data of different stages into the computer and let the computer involve in designing, analyzing, calculating, saving and sharing, which enables the effect of the design to be shown in different aspects at real time and to be compared and composed with the actual landscape. It allows the user to check the effect of the design and revise it at the same time, and finally produce the drawings of landscape design, including design drawings, effect drawings, schedule drawings and 3D graphics.

If the user wants to create an excellent design report with rich contents, he or she can make the preliminary designs special effects. The stages mentioned above are the basic steps of CAD landscape design. In such processes, the main task of designers is to create designs and drafting is of secondary status.
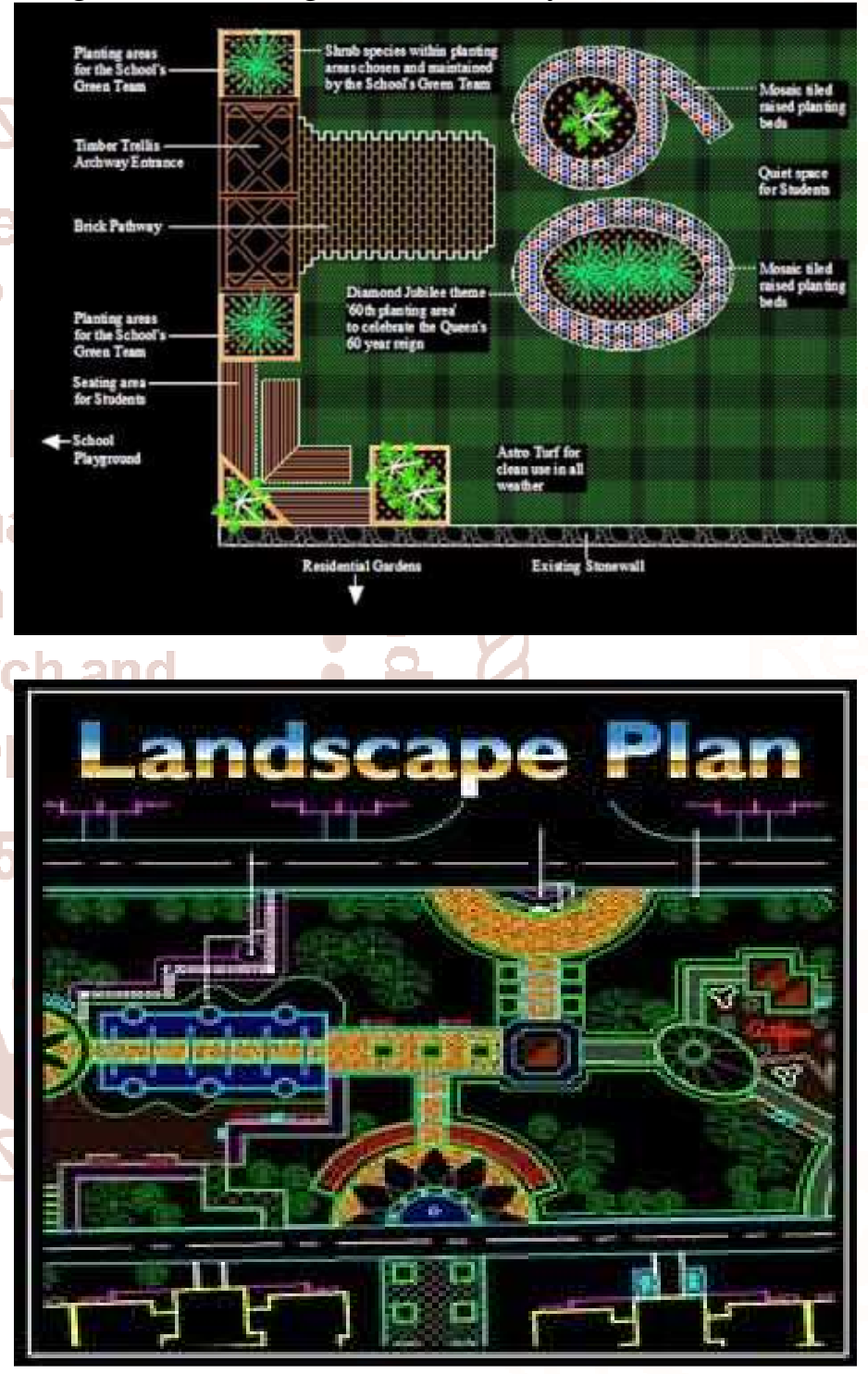

\section{CAD software programs for landscape design}

The currently widely used software programs in landscape design are as follows

1. HCAD. HCAD is a re-developed professional software program based on AutoCAD's platform, used in landscape design, landscape engineering and urban planning, ect. It's accessible, professional and able to develop extensions. All of which meet the 
needs of landscape design. This software program is practical and fit for Chinese landscape design because it's developed in accordance with China's standards and the protocols in this line of business. By using this software program, the user can increase the efficiency and improve the quality of the design. HCAD also provides Chinese menu for Chinese users. The layout of menu is clearer and enables the user to work fast and efficiently under a multiple document interface. Besides, its editing function is user-defined. Employment of Object ARX facilitates the perfect integration of the programming and the software. The object property manager of HCAD also enables the user to read and revise the parameters of user-defined objects at real time. HCAD is flexible in ways of design. There are several options of paths when a task is finished and either path can lead to the final completion of the task. The paths form an interconnected network as a whole. There is only a difference of distance but no dead ends.

\section{LANDCADD. LANDCADD is a software} program especially developed for landscape design and drafting by American corporation Eagle Point Software. It usually runs on graphic support platform like AutoCAD, but it has independent built-in platform. It's widely used in various countries because it's professional, complete and has powerful functions. It has become an authoritative software program in landscape design and drafting. LANDCADD consists of data acquisition, data transfer, node localization, measurement $\mathbb{N}$ and correction, surface modeling, site planning, site design, base plan, landscape design, design of irrigation systems, detailed drafting, quantitative extraction, plants database and visual simulation. These different functions are independent and complementary to each other. Although users work on different designs, LANDCADD will offer them series solutions.

LANDCADD has many advantages, powerful functions and improved system. But it's not perfect for Chinese users. Firstly, it doesn't provide matching establishment. It's only used as a software program but its matching establishment is not available, which is something shouldn't be neglected. Only the combination of these two can let it perform its various functions. Secondly, it has geographical restrictions. LANDCADD was initially developed in the United States and it's not fit for Chinese landscape design, unable to provide perfect demonstrations of Chinese landscape designs. Take plants, the core of landscape design, for example, the content of its plants database is mainly plants in North America and lacks Chinese plants, which burdens Chinese users a lot of extra work. Thirdly, it's very expensive. The high price and limitations make it difficult to become popular.

3. AutoCAD. AutoCAD is a commercial software application for computer-aided design (CAD) and drafting, developed and marketed by Autodesk, an American corporation. As widely used software for graphic design, it's accurate, accessible and has powerful functions. What's more, it's also available for Chinese, which makes it popular among Chinese landscape designers. For landscape design, AutoCAD is used to draft various kinds of graphics, 3D graphics and effect drawings. It not only increases efficiency but also is compatible with other design and planning programs and therefore enables the user to have access to shared information and data. It's especially useful in big projects. The working process of computer-aided design employed with AutoCAD is similar to traditional design.

The first step is to gather all the materials and information of the design object and design requirements. After having a comprehensive knowledge of design standards, it comes to the preliminary drafting. Then the user needs to work out the patterns, sizes of the elements in the draft and their functions to the object. When it's all finished, the user will build or download a suitable legend library for the established work. Finally it comes to the actual design on the compute.

AutoCAD's capabilities are insufficient to both enhance the performance of landscape design and finish the project ahead of plan. So it's necessary to have extensions on AutoCAD. And AutoCAD possesses the advanced ability of re-development and Auto LISP, the exclusive programming language. Besides, it's available for users to employ other advanced programming languages like $\mathrm{C}++$ to create the menu or functions they need.

4. 3DMAX. 3DMAX is a professional 3D computer graphics program for making $3 \mathrm{D}$ animations with powerful functions, providing a comprehensive modeling, animation, simulation, and rendering solution for motion graphics artists. It has rich materials, various lighting effects and advanced compositing editor. Comparing with AutoCAD, 
3DMAX has certain advantages in modeling, such as loft in three-dimensional paths and surface modeling, which are exactly what AutoCAD cannot provide. It's doing a great job in modeling projects including garden landscape sculptures and structures. Its particle system is particularly good at modeling fountains and running water.

In the field of 3D rendering, 3DMAX is currently an advanced core software program, providing a comprehensive modeling, animation, and rendering solution. In CAD landscape design, its static 3D rendering is mostly employed. 3DMAX is compatible with AutoCAD's DXF 3D models. After selecting the materials, setting up and lighting effects and environmental conditions, the user is able to create the design from a specific perspective, and the final rendering graphics can be saved in various forms. However, although it has rich material library and graphics library, the species, forms and structures of real landscape plants are more complicated, and it still cannot provide a vivid, comprehensive display of actual situation.

Currently, among various 3D graphics software programs, 3DMAX is widely used for its powerful functions. It's advanced in modeling, rendering and post production, but in terms of open structure and accurate drafting, it's not as good as AutoCAD.

\section{THE APPLICATION/OF VIRTUAL REALITY IN LANDSCAPE DESIGN \\ The concept of virtual reality}

Virtual reality is a computer technology that combines sensing and simulation. It creates an imagined world with computer, which can be felt with sensory experiences. This technology creates more realistic virtual reality for users and enables users to integrate with the environment. Virtual reality contains all kinds of information technology and is the latest product of computer science. It's different from traditional $\mathrm{CAD}$ and 3D animation. Virtual reality aims at creating an open, interactive environment that allows the user to interact with the object. By the concept, it has three basic features: immersion, interaction and imagination. All of them are in dispensable shown in below Figure.

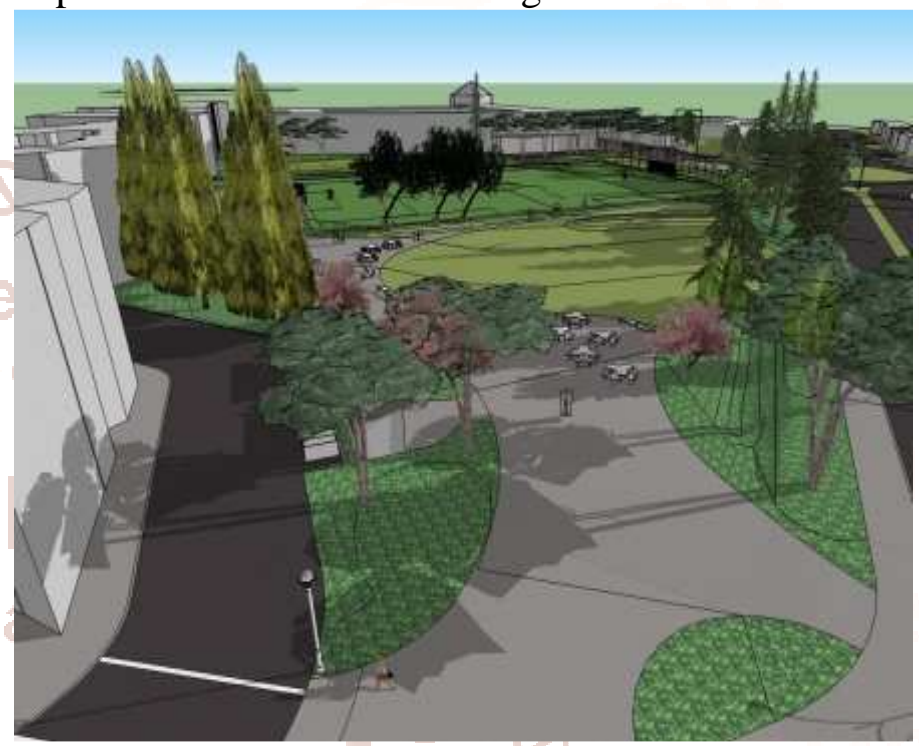

The process of employing virtual reality in landscape design

Virtual reality aims at creating a virtual scene, through which the designer can assess the design by walkthroughs, spot the problems and provide solutions. There are two major systems in virtual scenes: the modeling environment and the real time walkthrough environment shown in below figure.

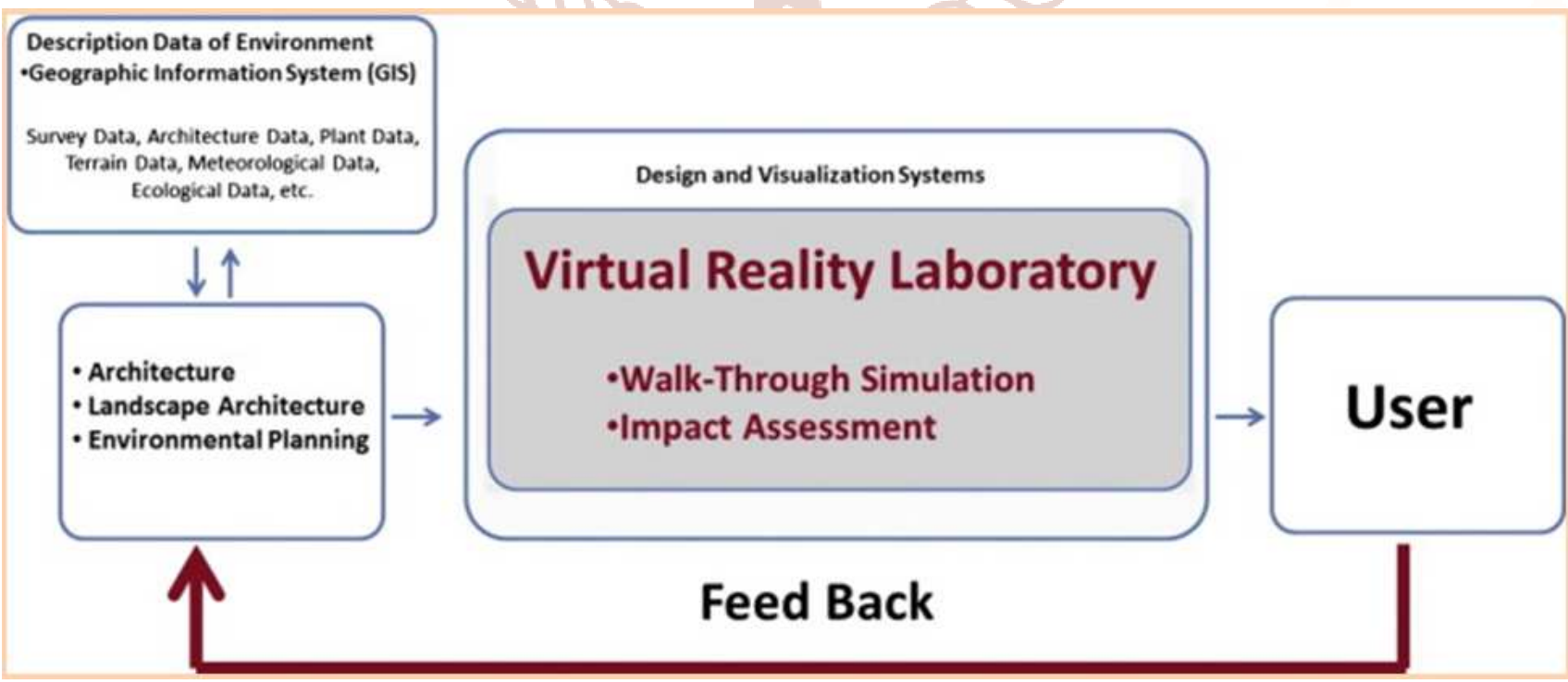


Confirming the virtual reality plan for the project

Different projects require different design processes and design methods. This essay takes an urban park as the project, with an area of $100000 \mathrm{~m} 2,1.2$ million scenes, which is a medium-sized project in virtual reality.

\section{Gathering and sorting out the information}

The first step is to sort out present modules according to the requirements of the project, then to gather and arrange planning maps, CAD drawings, pictures and drawings. This part is the most important in the whole process. The quality of the information determines the efficiency of the design, final results and at the same time avoids unnecessary repeated gathering.

\section{Scene modelling}

This part is the foundation of the whole process. The final effect depends on the modeling to a large extent. The scenes of the park are finished by both 3DMAX and AutoCAD. Graphics are firstly drafted by AutoCAD then input into 3DMAX for modeling. Then use Quest3D to arrange the elements. It finally comes the vivid 3D model.

\section{Plants modeling}

Plants require special attention in virtual reality due to its important role in landscape design. And its wide variation range is the biggest challenge in visualization. There are three methods for its modeling: surface texture, geometric model and holographic model. All of these three methods were made comparison and employed to model the plants.

\section{The advantages of virtual reality employed in landscape design}

The advantages of virtual reality are not only about it's being real time, interactive, but also because it provides what traditional methods cannot provide: information interactive interface. It allows the user to get into the 3D space. While personally experiencing it, the user can edit the elements in the design and change the 3D effects in real time. It provides powerful technical support for analyzing design plans and design innovations, features and relevant materials. Virtual reality has four major advantages: guaranteeing the accuracy of gathered information and its analysis; making design ideas into dynamic 3D experience; providing computer aided design throughout the whole process; systematizing the information.
In terms of the future of landscape design, virtual reality provides strong support for creating multidimensional space experience. The application scope of virtual reality in landscape design includes:

1. 3D experience of the space of the design;

2. Employing several methods for simulation;

3. Observing the environment from certain perspectives;

4. Editing the elements in the landscape design at real time;

5. A large amount of information exchange through multidimensional spac;

6. Helpful in making final decisions and increasing people's engagement.

\section{The future perspective of virtual reality employed} in landscape design

Even though it has been a short period since virtual reality was developed but it has achieved significant progress. However, its advancement has been overstated and needs further exploration. In fact, we should be consciously aware that the current stage still needs to be developed and has a long way to go to reach the expectant goal. The exploring areas are also far from being all-round. Besides, the 3D models created by computer are made of surfaces. The more complex the model is, more surfaces it needs. Therefore it requires high level of computer technology. But the current computer technology common designers' use does not meet the demands. The future perspective of CAD landscape design is quite bright: graphics will be faster and of better quality; input and output equipments will be more reasonable and suitable; processing abilities will be further improved. Living in an era of rapid development of science and technology, computer technology has become an indispensable part of human society. Being able to employ computer technology has become an essential requirement for landscape designers. And it's designers' duty to do so to create a new era for landscape design.

\section{CONCLUSION}

Judging from the discussion above, the current development of computer technology has brought 5858 Analysis of computer aided design employed in landscape design BTAIJ, 10(12) 2014 increasing benefits to landscape design and its statues is also on the rise. Computer technology makes up what traditional design methods cannot achieve, making landscape design faster, more convenient and more 
efficient. Therefore, the development of computer technology has explored broader space for landscape design. Landscape designers can use computer to perfect design plans, improve working efficiency and optimize the interests. CAD landscape design will have a bright future and become further integrated. How to employ computer technology will be a hot topic worth further exploration.

\section{REFERENCES}

1. Yang Meihua, Song Yuyang, Tayier; A Discussion of Effect Drawings drafted by Computer Aided Design [J]. Journal of Anhui Agricultural Sciences, 35(17), 5326-5327 (2007).
2. Liu Xiaozhao; Modern Information Technology's Application in Landscape [J]. Journal of Jiangsu Forestry Science \& Technology, 27, 1-3 (2000).

3. Xiao Huashun, Wang Jiaqiu, Zeng Siqi; Scientific Calculation's Visualization and its Application in Forestry [J]. World Forestry Research, 18(4), 1822 (2005).

4. Guo Jisong; Computer Aided Design's Employment in Landscape Design [J]. China Computer \& Communication, 03, 101 - 104 (2010).

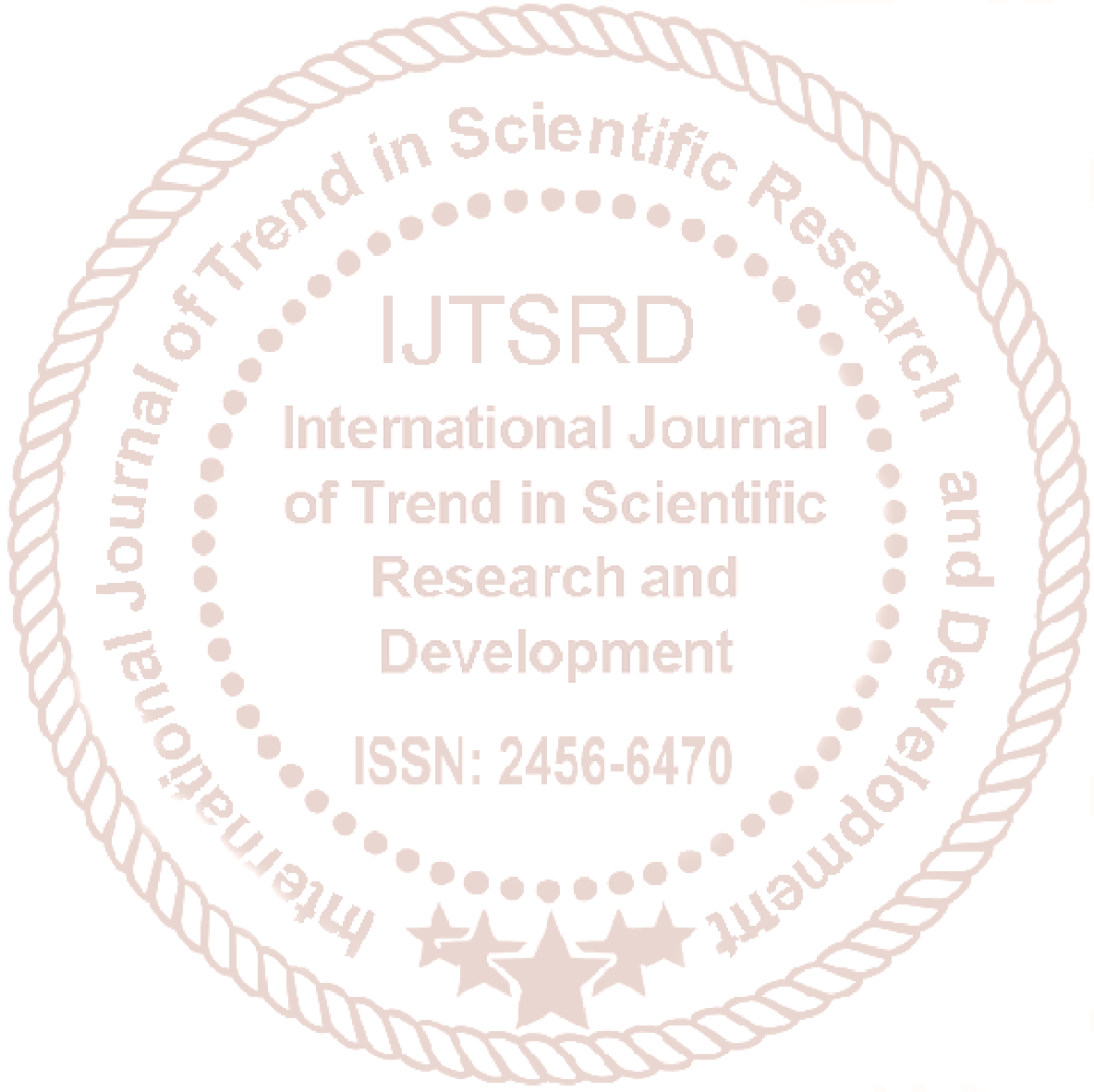

